Revue des sciences de l'eau

REVUE DES

Journal of Water Science

S C I E N CES

DE L'EA U

\title{
Modélisation du transport de solutés dans la zone non saturée du sol
}

Modeling of solute transport in the vadose zone

\section{Vauclin}

Volume 7, numéro 1, 1994

URI : https://id.erudit.org/iderudit/705191ar

DOI : https://doi.org/10.7202/705191ar

Aller au sommaire du numéro

Éditeur(s)

Université du Québec - INRS-Eau, Terre et Environnement (INRS-ETE)

ISSN

0992-7158 (imprimé)

1718-8598 (numérique)

Découvrir la revue

Citer cet article

Vauclin, M. (1994). Modélisation du transport de solutés dans la zone non saturée du sol. Revue des sciences de l'eau / Journal of Water Science, 7(1),

81-102. https://doi.org/10.7202/705191ar

\section{Résumé de l'article}

On présente une analyse critique des modèles décrivant les transferts d'eau et de substances chimiques dans les sols partiellement saturés en eau. Pour la clarté de l'exposé, ces derniers sont classés en modèles déterministes (mécanistes et fonctionnels) et stochastiques. Les avantages et limites de ces différentes approches sont discutés. Finalement quelques recommandations et voies de recherche relatives à ce domaine sont suggérées. 


\title{
REVUE - ÉTAT DE L'ART
}

\section{Modélisation du transport de solutés dans la zone non saturée du sol}

\author{
REVIEW - STATE OF THE ART
}

\author{
Modeling of solute transport \\ in the vadose zone
}

M. VAUCLIN1

Reçu le 7 juin 1993, accepté le 15 novembre 1993*.

\section{SUMMARY}

The importance of the unsaturated (vadose) zone as an inextricable part of the hydrologic cycle has long been recognized. Theoretical and experimental studies on both water flow and solute transport in this zone have been further motivated by attempts to manage the root zone of agricultual soils optimally as well as by concerns about soil and groundwater pollution. These studies have greatly increased our conceptual understanding of the many complex and interactive physical, chemical and microbiological processes operating in the unsaturated zone. They have also led to a large number of models which vary widely in their conceptual approach and degree of sophistication, and are strongly influenced by the environment, training and preoccupations of their developers.

A number of conceptual modek for solute transport in partially saturated soils are reviewed and categorized. A key distinction is made between deterministic models which assume that a system behaves in such a way that the cocurrence of a given set of events leads to a uniquely definable outcome, and stochastic models, which presuppose the outcome to be uncertain and are structured to account for this uncertainty. A second main distinction is between mechanistic and functional modek. Mechanistic implies that the model takes into account the most fundamental mechanisms of the processes, as presently known and understood (e.g. Darcy's law for water now, combination of mass-flow and diffusion-dispersion mechanisms for solute transport). The term functional refers to models that incorporate simplified treatments of solute and water and make no claim to fundamentality. However, their use requires less input data and computer expertise as compared to mechanistic models.

Additionally, it may be useful to distinguish between models that are primarily research tooks (most of the mechanistic models) developed either to aid the testing of assumptions or to contribute to better understanding of the physical processes, and those (functional models) that are mainly useful as guides to the management of soil and water resources.

1. Laboratoire d'Etude des Transferts en Hydrologie et Environnement (INPG, UJF, CNRS, URA 1512), BP 53, 38041 Grenoble Cedex 9, tél. (33) 76825056 .

* Les commentaires seront reçus jusqu'au 31 octobre 1994. 
Although no attempt is made here at an exhaustive litterature review, the main features, the strengths and weaknesses of these approaches are presented and discussed. This analysis and other reviews published in recent years have revealed progress in many areas. Investigators have uncovered a number of inadequacies with existing models of soil transport processes and have made significant steps toward a better understanding of these phenomena. Some new research areas such as biodegradation modeling, immiscible phase transport, water and solute transfers in structured or swelling soils, and multi-interative ion transport are emerging.

A number of challenges still remain for both the theoretician and the practitioners. They include: i) how best to deal with preferential water flow and transport, ii) how best to modet the effects of local and regional spatial and temporal variabilities of soil hydraulic properties on solute transport, iii) how to couple multi-component geochemical submodels efficiently with available unsaturatedsaturated flow modes, iv) how to improve field methods for estimating vadose zone transport parameters and v) how best to predict the long-term consequences of short-term management decisions.

It is apparent that the complexity and variety of the physical processes have led to increasing specialization within the area of transport modeling. Soil physicists, soil chemists, soil microbiologists and agronomists have the propensity to limit their consideration and vision to their respective disciplines. A natural consequence of this specialization has been the evolution of scientific jargon specific to each subdiscipline. This may be overcome by reinforcing interdisciplinary cooperation among investigators, by traîning of students both at the graduate and postgraduate levels and by encouraging topical workshops and publications in interdisciplinary journals. Another general observation gleaned from this review is that very few solute transport models have been exhaustively tested under field conditions. Indeed, the quantitative criteria for validating models do not seem to be clearly identified or universally recognized. It appears very important that such criteria should be established and used to make an objective comparison of the abilities of the various types of model to simulate the results of field experiments. Without such tests and without comparisons between models there is a risk that disagreements between the predictions of different models and the resulting confusion could greatly diminish the usefulness of modeling techniques. While computer codes excalate in number as pressures mount for improved managements strategies it is time for asking the question: should the scientific community continue to develop more and more sophisticated general or even spectific models or should it put an emphasis on field experiments ? Obtaining an answer will probably be of great importance in the near future. As a matter of fact, because of decreasing computer costs and relative increase in the cost of carefully designed field experiments, there is a worldwide trend to * observe the water and solute movements through computer screens " ? The modelers should be aware that without reliable estimates of the input parameters as well as in-situ validation their models will appear more as intellectual games of academic interest rather than as took to help the practitioners in their management decisions.

Key-words : water, soil, solutes, deterministic model, stochastic models.

\section{RÉSUMÉ}

On présente une analyse critique des modèles décrivant les transferts d'eau et de substances chimiques dans les sols partiellement saturés en eau. Pour la clarté de I'exposé, ces derniers sont classés en modèles déterministes (mécanistes et fonctionnels) et stochastiques. Les avantages et limites de ces différentes approches sont discutés. Finalement quelques recommandations et voies de recherche relatives à ce domaine sont suggérées.

Mots clés : eau, sol, solutés, modèle déterministe, modele stochastique. 


\section{1 - INTRODUCTION}

La description et la prévision des transferts de masse (eau, substances chimiques) et de chaleur dans les couches superficielles du sol revêtent une importance certaine dès qu'il s'agit de résoudre des problèmes concrets liés aux sciences environnementales telles que l'hydrologie, l'hydrogéologie, l'agronomie, la climatologie, le génie civil et sanitaire, etc. C'est en effet dans ce domaine physique appelé classiquement zone non saturée, interagissant d'une part avec l'atmosphère, d'autre part avec les aquifères, que se situent les réserves en eau et en substances nutritives pour les plantes, qu'a lieu l'essentiel des transferts conduisant à l'évapotranspiration, l'infiltration et la recharge des nappes à surface libre. Il est également le siège de transformations bio-physico-chimiques des composés minéraux et organiques. L'euphorie passée que cette tranche de sol puisse constituer un "filtre vivant" capable de retenir et de dégrader en totalité et de façon pérenne les substances potentiellement polluantes, voire toxiques qui lui sont appliquées, a fait place ces dernières années à une réalité douloureuse : leur migration vers la biosphère et l'environnement plus profond est une évidence à la probabilité d'occurrence importante.

La compréhension des phénomènes est rendue complexe par le fait qu'il s'agit d'étudier des processus couplés, essentiellement non linéaires dans un milieu polyphasique (eau-air-solide) susceptible de se déformer et dans lequel les concentrations en fluides varient dans le temps et l'espace sous l'action conjuguée de variations d'énergie mécanique et thermique avec la possibilité de changements de phase et d'échanges physico-chimiques entre phases. Lorsqu'on aborde l'étude expérimentale et la modélisation des transferts dans un tel milieu hiérarchisé, trois échelles, correspondant à des niveaux d'analyse et de préoccupations différents doivent être considérées :

a) l'échelle microscopique (le pore) supposée grande devant les dimensions moléculaires. A cette échelle, les grandeurs locales présentent de larges fluctuations. Chaque phase peut être considérée comme un milieu continu, et le milieu poreux comme un ensemble de milieux continus plus ou moins imbriqués. C'est à cette échelle qu'il convient de se placer pour la compréhension fine des mécanismes fondamentaux mis en jeu.

b) l'échelle macroscopique : la colonne de laboratoire. La complexité géométrique de l'espace poral associée aux difficultés que posent la définition précise de la topologie des différentes phases et la métrologie des variables et paramètres font que la description microscopique ne peut être mise en pratique sans un changement d'échelle dont l'étape essentielle conduit à introduire le concept de Volume Elémentaire Représentatif. II permet d'établir une équivalence entre le milieu réel dispersé et un milieu fictif continu. A cette échelle, les phénomènes sont décrits par des équations dont les variables et les paramètres sont représentatifs de grandeurs moyennes au sein du continuum de milieu poreux. 
c) l'échelle mégascopique : le terrain. L'extension de l'approche précédente au milieu naturel se heurte à de sérieuses limitations :

i) un sol n'est jamais uniforme et homogène. Ses propriétés physicochimiques varient d'un "point macroscopique" à un autre. A titre d'illustration, le tableau 1 recense quelques résultats certes non exhaustifs, mais montrant clairement la grande variabilité spatiale (des coefficients de variation supérieurs à $300 \%$ ne sont pas rares) des propriétés hydro-dispersives des sols et des concentrations et ce, sur des surfaces allant de $100 \mathrm{~m}^{2}$ à $150 \mathrm{ha}$. On notera la tendance à une distribution log-normale $(R=$ Mode/moyenne $\neq 1)$ pour l'ensemble de ces variables. Etudier et modéliser les transferts nécessitent alors d'utiliser des concēpts statistiques et géostatistiques pour analyser cette variabilité et impliquent bien souvent d'ajouter à l'aspect mécaniste des équations, une description stochastique des paramètres pertinents.

ii) l'aspect statistique n'implique pas nécessairement que ces propriétés soient aléatoirement distribuées dans l'espace. Elles présentent bien souvent une structure horizontale et/ou verticale qu'il est important d'analyser et de prendre en compte aux plans de la reconnaissance du milieu et de sa modélisation.

iii) dans l'environnement naturel, le caractère aléatoire des conditions aux limites renforce l'aspect stochastique de la démarche.

Tableau 1 Exemples de variabilité observée. $\sigma$ est l'écart type, $R=$ Mode/moyenne. $\mathrm{N}$ est le nombre d'observations nécessaires pour estimer la valeur moyenne m à $10 \%$ près, au seuil de probabilité de $95 \%$.

Table 1 Observed variability. $\sigma$ is the standard deviation, $R=$ Mode/mean. $N$ is the number of observations required to estimate the mean value, $m$ with a specified relative error of $10 \%$ at the $95 \%$ confidence level.

\begin{tabular}{|c|c|c|c|c|c|}
\hline Variables & m & $\sigma$ & $\begin{array}{c}\text { Echantlllon } \\
\text { Observation/aire }\end{array}$ & $\mathbf{R}=\mathbf{M} / \mathbf{m}$ & N \\
\hline \multicolumn{6}{|l|}{ Perméabilité saturée $(\mathrm{cm} / \mathrm{l})$} \\
\hline - NIELSEN et al., 1973 & 25,8 & 64,8 & $120 / 150$ ha & 0,19 & 750 \\
\hline - Vou, 1986 & 36,3 & 135,3 & $101 / 2$ ha & 0,027 & 5290 \\
\hline \multicolumn{6}{|l|}{ Facteur d'écheile } \\
\hline - W/RRICK ef al., 1977 & 1,06 & 1,81 & $120 / 150$ ha & 0,13 & 1110 \\
\hline -RUSSO-BRESLER, 1980 & 1 & 0,73 & $120 / 0,8$ ha & 0,68 & 112 \\
\hline $\begin{array}{l}\text { - Vauclin et al., } 1983 \\
\text { Coefficlent de dispersion }\left(\mathrm{cm}^{2} / \mathrm{i}\right)\end{array}$ & 1 & 0,47 & $23 / 1$ ha & 0,72 & 92 \\
\hline $\begin{array}{l}\text { - Biggah-NiELSEN, } 1976 \\
\text { Vitesse de pore }(\mathrm{cm} / \mathrm{i})\end{array}$ & 367 & $1,410^{3}$ & $359 / 150$ ha & 0,011 & 7150 \\
\hline $\begin{array}{l}\text { - Biggar-NieLsen, } 1976 \\
\text { Diffusivité gazeuse }\left(\mathrm{m}^{2} / \mathrm{l}\right)\end{array}$ & 44,2 & 85,4 & $359 / 150$ ha & 0,098 & 1400 \\
\hline $\begin{array}{l}\text { - GruNdMANK at al., 1988 } \\
\mathrm{NO}_{3}^{-}(\mathrm{ppm})\end{array}$ & 0,15 & 0,16 & $67 / 90 m^{2}$ & 0,31 & 448 \\
\hline - TABOR et al., 1985 & 13,6 & 4,24 & $49 / 13$ ha & 0,87 & 37 \\
\hline $\begin{array}{l}\text { - Grundmann of al., } 1988 \\
\text { Flux de } \mathrm{N}_{2} \mathrm{O}\left(\mathrm{mg} / \mathrm{m}^{2} \mathrm{~h}\right)\end{array}$ & 427 & 246,4 & $67 / 90 \mathrm{~m}^{2}$ & 0,65 & 130 \\
\hline - FOLORUNSO-ROLSTON, 1984 & 0,63 & 1,77 & $36 / 90 \mathrm{~m}^{2}$ & 0,037 & 3055 \\
\hline - GRUNDMANn et al., 1988 & 0,42 & 0,63 & $67 / 90 \mathrm{~m}^{2}$ & 0,17 & 876 \\
\hline
\end{tabular}


Dans le cadre de cette présentation, il est évidemment exclu et prétentieux de traiter l'ensemble de ces problèmes dans toute leur généralité. On se propose ici de faire le point sur la modélisation mathématique du transport de solutés dans la zone non saturée des sols, en mettant l'accent plus sur les verrous actuels que sur les succès passés.

Dans la suite, un modèle sera considéré comme une image schématique (et non comme un exemple à suivre) d'une réalité physique complexe. II sera susceptible de se mettre sous la forme générale suivante:

$$
\text { - } Y=F\left(X_{i}, a_{j}\right)+\varepsilon
$$

où $X_{i}$ et $Y$ sont les vecteurs des variables d'entrée et de sortie respectivement, $a_{\mathrm{j}}$ les paramètres et $\varepsilon$ l'erreur commise en assimilant l'objet d'étude à son image. Elle permet une classification (il en existe d'autres) des modèles :

- un modèle conceptuel (de type mécaniste ou fonctionnel) correspond à une fonction $F$ fondée sur une certaine connaissance (et pas nécessairement certaine) des processus impliqués.

- lorsque $F$ résuite d'expériences, il s'agit d'un modèle empirique.

- quelle que soit la nature de la fonction $F$, considérer les variables d'entrée et/ou les paramètres comme appartenant à des Fonctions Aléatoires conduit à un modèle stochastique. Dans le cas contraire, il s'agit d'un modèle déterministe.

Selon cette terminologie, on étudiera plus particulièrement les modèles conceptuels déterministes et stochastiques en mettant l'accent sur l'aspect mécaniste, dans la mesure où ce dernier constitue la moins mauvaise approche pour une description des principaux processus mis en jeu.

Le lecteur intéressé, mais frustré par la concision du texte a la possibilité de se reporter pour plus de détails aux articles cités dans la liste des références.

\section{2 - MODĖLES DÉTERMINISTES MÉCANISTES}

\section{1 Équations de base}

Très classiquement, le transport isotherme d'une espèce chimique (i) est modélisé, à l'échelle macroscopique, par l'équation suivante :

$$
\frac{\partial\left(p_{i}\right)}{\partial t}+\frac{\partial\left(\theta C_{i}\right)}{\partial t}=\frac{\partial}{\partial z}\left\{\theta D_{i}(\theta, q) \frac{\partial C_{i}}{\partial z}\right\}-\frac{\partial}{\partial z}\left(q C_{i}\right)+\sum_{j=1}^{n} \phi_{i, j}\left(C_{i}, S_{i}\right)+A_{c, i}(z, t)
$$

(a)

(b)

(c)

(d)

(e)

où $C_{i}$ et $S_{i}$ sont les concentrations de la substance (i) associées aux phases fluide et solide respectivement, $D_{i}$ est le coefficient apparent de dispersion hydrodynamique de l'élément (i) ; $\rho$ est la masse volumique du sol sec, $\theta$ la teneur volumique en eau du sol, $\sum_{j=1}^{n} \phi_{i, j}\left(C_{i}, S_{i}\right)$ représente les consommations 
de l'espèce (i), et q la densité de flux volumique donnée par la loi de Darcy généralisée:

$$
q=-K(h)\left(\frac{\partial h}{\partial z}-1\right)
$$

dans laquelle $\mathrm{h}$ est la pression de l'eau du sol (exprimée en hauteur ds colonne de liquide) qui dépend de $\theta ; \mathrm{K}(\mathrm{h})$ est la conductivité hydraulique : $z$ est l'axe des profondeurs, orienté positivement vers le bas.

Dans le cas d'un écoulement transitoire, ce flux hydrique résulte de la solution de l'équation:

$$
C(h) \frac{\partial h}{\partial t}=\frac{\partial}{\partial z}\left\{K(h)\left(\frac{\partial h}{\partial z}-1\right)\right\}+A_{w}(z, t)
$$

où $C(h)=d \theta /$ dh est la capacité capillaire du sol, et $A_{w}$ représente un terme $d E$ source ou de puits (par exemple le prélèvement d'eau par le système racinaire).

Chaque terme de l'équation (2) est représentatif d'un mécanisme distinct :

- le premier terme (a) décrit le taux de passage de l'espèce (i) de la phase liquide à la phase solide et vice-versa. II représente les processus d'adsorption-désorption et d'échanges ioniques ;

- le terme (b) rend compte du taux de variation (positive ou négative) de l'espèce (i) dans la phase liquide ;

- le terme (c) décrit le transfert dispersif associant la diffusion moléculaire (ou ionique) au mélange mécanique provoqué par les variations spatiales de la vitesse de l'écoulement à l'échelle microscopique. Le coefficient de dispersion $D$, observé à l'échelle macroscopique est donc censé intégrer et moyenner les fluctuations microscopiques ;

- le terme (d) représente le transfert convectif, l'élément (i) étant transporté par le flux d'eau, $\mathbf{q}$;

- le terme (e) prend en compte les termes sources de l'espèce (i) :

- le dernier terme (f) représente l'éventuel prélèvement de la substance (i) par le système racinaire.

A travers les termes (a) et (e), l'équation (2) décrivant le transport est couplée à différents sous-modèles chimiques brièvement décrits ci-dessous.

\subsection{Réactions chimiques}

Le terme (e) de l'équation (2) peut inclure différents processus chimiques tels que les réactions de précipitation/dissolution, les oxydo-réductions, les transformations d'origine microbiologique d'une espèce en une autre, la décroissance radioactive, etc. Mis à part ce demier phénomène, ce terme est en général difficile à modéliser. D'une part l'identification des mécanismes n'est pas toujours évidente, et d'autre part les interactions avec d'autres facteurs de l'environnement ne sont pas que locales. Cela conduit bien souvent à introduire des relations paramétriques pseudo-empiriques. Ainsi, ce terme est très souvent approché par une expression du type: 


$$
\sum_{j=1}^{n} \phi_{i, j}=-\mu_{1}^{i} \theta C_{i}-\mu_{s}^{i} \rho S_{i}+\gamma_{1}^{i} \theta+\gamma_{s}^{i} \rho
$$

où $\mu_{1}^{i}$ et $\mu_{s}^{i}$ caractérisent les taux de disparition de l'élément (i) dans les phases liquide et solide respectivement, $\gamma_{1}^{i}$ et $\gamma_{s}^{i}$ étant les taux de production de liquide et de solide. Ces différents coefficients doivent faire l'objet de détermination expérimentale.

\subsection{Modèles d'adsorption-dẻsörption}

Le premier terme de l'équation (2) décrit le taux avec lequel une substance chimique interagit ou échange avec la phase solide du sol. Sa modélisation est l'objet de nombreuses études et débats. Des hypothèses d'équilibre et de non équilibre local sont utilisées pour décrire ces processus.

a) L'hypothèse d'équilibre local (LEA) conduit à considérer ces processus comme instantanés, comparés aux temps caractéristiques de la convection et de la dispersion. Ils sont décrits par des isothermes d'équilibre $S$ (C) de différents types [linéaire, Freundlich, Langmuir, etc. (voir BOLT, 1979)] et présentant bien souvent une hystérésis entre l'adsorption et la désorption, notamment pour les substances organiques (VAN GENUCHTEN et al.,1974); CALVET et al., 1980). Le modèle probablement le plus utilisé est une simple relation linéaire entre $S$ et $C$ :

$$
\mathrm{S}=\mathrm{K}_{\mathrm{d}} \mathrm{C}
$$

où $K_{d}$ est appelé coefficient de distribution.

Bien que cette forme soit très agréable à traiter, notamment dans les modèles de transport en régime hydrique permanent (voir VAN GENUCHTEN et ALVES, 1982, pour les nombreuses solutions analytiques correspondantes) son domaine de validité est restreint (VALOCCHI, 1984) à une gamme réduite de concentration.

b) L'hypothèse de non équilibre local (NLEA) est très souvent invoquée pour expliquer des discordances importantes entre les résultats de modèles fondés sur l'équilibre local et les observations. Elle a conduit au développement de deux types de modélisations.

i) ceux fondés sur la prise en compte d'une cinétique chimique :

- à un site (i.e. LAPIDUS et AMUNDSON, 1952) :

$$
\frac{\partial \mathrm{S}}{\partial \mathrm{t}}=\alpha(\mathrm{KC}-\mathrm{S})
$$

- ou à deux sites (i.e. VAN GENUCHTEN et al., 1990) :

$$
S_{1}=F K C \text { et } \frac{\partial S_{2}}{\partial t}=\alpha\left[(1-F) K C-S_{2}\right]
$$

où $\mathrm{F}$ est la fraction des sites atteignant instantanément l'équilibre. 
ii) ceux fondés sur l'existence physique d'une distribution bi-modale de vitesse de pore conduisant à lintroduction du concept de fractions d'eau mobile et immobile avec cinétique d'échange (VAN GENUCHTEN et WIERENGA, 1974 ; GAUDET et al., 1977, etc.).

L'applicabilité de ces différents concepts a été largement démontrée pour les milieux réactifs ou non, et pour une grande variété de traceurs. Leur utilisation a permis d'améliorer le caractère prédictif des modèles de transport fondés uniquement sur les processus de convection et dispersion avec ou sans l'hypothèse d'équilibre local. Comment pourrait-il du reste en être autrement dès lors que le oombre de paramètres augmente de façon significative?

\subsection{Avantages et inconvénients de l'approche mécaniste}

Dans la mesure où les modèles mécanistes essaient de formaliser le maximum de processus, ils constituent d'excellents outils de recherche permettant notamment d'étudier en détail, les interactions physico-chimiques dans les sols, et de conduire de manière rigoureuse des études de sensibilité à tel ou tel phénomène ou paramètre. Néanmoins, ils présentent certaines limitations, brièvement rappelées ci-dessous.

\section{a) Détermination des paramètres}

La résolution numérique (différences finies, éléments finis, etc.) ou analytique (dans certains cas bien particuliers et en général très éloignés de la réalité de terrain) des équations requiert la connaissance préalable des paramètres dont le nombre dépend évidemment du degré de sophistication des modèles eux-mêmes. La difficulté d'obtention directe de la plupart d'entre eux fait qu'ils sont obtenus le plus souvent par ajustement du modèle à des données expérimentales (laboratoire ou terrain mais obtenus en conditions contrôlées voire artificielles) en utilisant des procédures d'optimisation. Bien que ces demières deviennent de plus en plus performantes, elles posent bien entendu le problème de l'unicité de la solution trouvée. Cela conduit à conférer aux modèles un caractère semi-empirique lié au mode d'obtention des paramètres phénoménologiques.

\section{b) Validation des modèles}

Un examen de la littérature montre qu'en général la pertinence des paramètres identifiés à partir des solutions numériques ou analytiques est rarement évaluée sur d'autres données que celles qui sont utilisées dans la procédure d'identification elle-même! De plus, alors que les modèles simulent les flux d'eau et de matière, ils sont "validés " bien souvent sur des mesures de concentrations qui ne sont pas nécessairement les meilleurs indicateurs des flux au travers de la zone non saturée, en raison notamment de l'existence d'écoulements préférentiels (présence de macropores d'origine structurale ou biologique; phénomènes d'instabilité hydrodynamique conduisant à des digitations, ...). 


\section{c) Utilisation des modèles en conditions naturelles}

La variabilité spatiale des sols et notamment de leurs propriétés hydrodynamiques conduit à douter de la valeur prédictive de modèles fondés sur l'hypothèse d'unicité des relations vitesse de pore-teneur en eau-coefficient de dispersion. Cet aspect, couplé aux difficultés expérimentales d'obtention insitu des paramètres phénoménologiques tels que la relation conductivité hydraulique-teneur en eau, a stimulé d'autres approches abordées succinctement ci-dessous.

\section{3 - MODĖLES DÉTERMINISTES FONCTIONNELS}

Contrairement aux modèles précédents qui sont essentiellement fondés sur la notion de vitesse ou de flux de matière, les modèles dits " fonctionnels" sont de type capacitif : le sol est assimilé à un réservoir défini par deux humidités caractéristiques : le point de flétrissement et la capacité au champ.

Selon ADDISCOTT et WAGENET (1985), deux classes de modèles fonctionnels peuvent être considérées :

\subsection{Les modèles continus}

Dans l'équation du transport, la diffusion et la dispersion sont négligées de façon à ne considérer que le transport convectif (écoulement piston) qui permet une détermination triviale du pic de concentration autour duquel on peut éventuellement surimposer ces deux effets (DE SMEDT et WIERENGA, 1978 ; ROSE et al., 1982).

Bien que ce type de modèle, partiellement ou totalement analytique, ait été utilisé avec un certain succès pour simuler le devenir de créneaux de concentration appliqués à la surface d'un sol (DE SMEDT et WIERENGA, 1978 pour $\mathrm{Cl}^{-}$; ROSE et al., 1982 pour ${ }^{15} \mathrm{~N}$; CAMERON et al., 1982 pour ${ }^{36} \mathrm{Cl}$ ), il présente de sérieuses limitations dès lors que le profil vertical de sol est hétérogène ou contient déjà une concentration initiale. De plus, à une exception près (SCOTTER, 1978), il n'a pas été utilisé pour des substances interactives avec la phase solide.

\subsection{Les modèles discrets}

Des considérations chromatographiques (REINIGER et BOLT, 1972 ; VILLERMAUX, 1981) ont été utilisées pour simuler le lessivage des sols. II s'agit ici de diviser le sol en une succession de cellules horizontales dans lesquelles et entre lesquelles les différents processus physiques, chimiques voire biologiques sont pris en compte. Le principe de conservation de la masse appliqué à l'eau êt aux substances chimiques conduit en général à une succession d'équations algébriques dont la résolution est aisée. 
Cette approche apparaît particulièrement pertinente dans le cas du transport simultané de plusieurs espèces chimiques (VALOCCHI et al., 1981; SARDIN et al., 1986) ou de substances fortement interactives avec la phase solide (FRISSEL et POELSTRA, 1967). Alors qu'elle est classiquement mise en œuvre en génie chimique, elle semble peu connue des hydrologues, physiciens et chimistes des sols.

\subsection{Avantages et inconvénients}

Ces modèles outre le fait qu'ils ont l'avantage de présenter une structure mathématique simple, n'utilisent qu'un nombre réduit de données d'entrée et de paramètres dont la plupart peuvent être obtenus sans procédure d'identification. Leur utilisation au terrain est facilitée par le fait que les paramètres "capacitifs" apparaissent moins variables que les paramètres " conductifs ". En revanche, leur conception ne leur permet pas de contribuer à une amélioration des connaissances sur les processus fondamentaux. Ils ne peuvent prévoir l'impact de modifications du milieu physique sur les processus de transport.

\section{4 - MODÈlES STOCHASTIQUES}

Il est bien connu depuis de très nombreuses années que les propriétés physico-chimiques locales des sols présentent de larges fluctuations spatiales. La nature, la modélisation de cette variabilité et les problèmes d'échantillonnage qui lui sont associés ont été abondamment décrits dans la littérature (WARRICK et NIELSEN, 1980 ; MAC BRATNEY et WEBSTER, 1983 ; VAUCLIN, 1983, et beaucoup d'autres...). Cette situation a conduit à considérer les phénomènes de transferts comme des processus essentiellement erratiques susceptibles d'une quantification par des modèles stochastiques que l'on peut regrouper en deux catégories : i) les modèles mécanistes, ii) les modèles empiriques type Fonction de Transfert.

\subsection{Modèles stochastiques, mécanistes}

Dans cette approche, les propriétés hydrodynamiques sont assimilées à des Fonctions Aléatoires qui, introduites dans un modèle mécaniste conduisent à exprimer la solution d'un problème de transport en termes probabilistes donc in-fine de risques.

lllustrons la démarche sur l'exemple relativement simple du transport d'un soluté non réactif dans un sol partiellement saturé :

i) à l'échelle de la colonne de laboratoire, admettons la validité du schéma classique issu des équations (2) et (4) : 


$$
\begin{gathered}
\theta \frac{\partial C}{\partial t}=\frac{\partial}{\partial z}\left\{\theta D(\theta, q) \frac{\partial C}{\partial z}\right\}-q \frac{\partial C}{\partial z} \\
C(h) \frac{\partial h}{\partial t}=\frac{\partial}{\partial z}\left\{K(h)\left(\frac{\partial h}{\partial z}-1\right)\right\}
\end{gathered}
$$

ii) à l'échelle du milieu naturel, considérer par exemple que la conductivité hydraulique est un processus stochastique homogène, ergodique $K(h, \omega)$ de loi de probabilité marginale $f_{K}$, implique que le flux de Darcy (Eq. 3), la vitesse de pore, le coefficient de dispersion hydrodynamique $D$ sont également des processus stochastiques, qui introduits dans les équations (8) conduisent à :

$$
\begin{gathered}
\theta(\omega) \frac{\dot{\partial} C(\omega)}{\partial t}=\frac{\partial}{\partial z}\left\{\theta(\omega) D(\omega) \frac{\partial C(\omega)}{\partial z}\right\}-q(\omega) \frac{\partial C(\omega)}{\partial z} \\
C(\omega) \frac{\partial h(\omega)}{\partial t}=\frac{\partial}{\partial z}\left\{K(\omega)\left(\frac{\partial h(\omega)}{\partial z}-1\right)\right\}
\end{gathered}
$$

La résolution de ces équations couplées aux conditions initiales et aux limites pour toutes les réalisations possibles de $K(\omega)$ permet d'obtenir, au moins en théorie les évolutions spatio-temporelles des fonctions aléatoires $h(z, t, \omega)$ et $C(z, t, \omega)$ dont les moments statistiques peuvent être calculés par:

$$
\begin{aligned}
& E\left\{h^{n}(z, t, \omega)\right\}=\int h^{n}(z, t, \omega) t_{k}(s) d s \\
& E\left\{C^{n}(z, t, \omega)\right\}=\int C^{n}(z, t, \omega) t_{k}(s) d s
\end{aligned}
$$

Alors que l'équation stochastique de Richards (Eq. 9b) a fait l'objet de nombreuses études (WARRICK et al., 1977 ; VAUCLIN et al., 1983 ; MANTOGLOU et GELHAR, 1987, etc.) notamment à travers la mise en facteur d'échelle des caractéristiques hydrodynamiques, l'équation du transport a relativement peu retenu l'attention à l'exception des modèles développés par BRESLER et DAGAN $(1979,1981,1983)$ qui restent encore à l'heure actuelle les plus représentatifs de cette approche.

L'ensemble de ces résultats actuellement disponibles (assez nombreux pour l'hydrodynamique, plus rares pour le transport) tend à montrer la non existence de "propriétés effectives" capables de caractériser un milieu homogène équivalent au milieu réel pour lequel la solution d'un problème hydro-dispersif en milieu non saturé serait identique à la moyenne des solutions du même problème en milieu hétérogène.

Une telle approche fournit un cadre conceptuel très intéressant et prometteur pour évaluer les effets de la variabilité. Elle présente l'avantage d'exprimer la solution d'un problème d'écoulement en termes probabilistes, donc de risques. On perçoit là l'intérêt pratique pour l'aménageur, le décideur. Néanmoins, dans la mesure où elle repose sur certaines hypothèses peu ou pas encore suffisamment validées expérimentalement, elle doit cependant être considérée au stade actuel de son développement, comme une tentative d'appréhension des impacts de la variabilité sur les transferts couplés eauéléments chimiques. 


\subsection{Fonctions de Transferts}

De façon très schématique, il s'agit d'établir une relation stochastique de la forme :

$$
Q_{\text {out }}(t)=\int_{0}^{t} g\left[\left(t-t^{\prime}\right) / t^{\prime}\right] Q_{\text {in }}\left(t^{\prime}\right) d t^{\prime}
$$

entre l'entrée $Q_{\text {in }}(t)$ et la sortie $Q_{\text {out }}(t)$ d'un volume de contrôle. Dans l'équation (11), $\mathrm{g}\left(\tau / \mathrm{t}^{\prime}\right)$ est la densité de probabilité des temps de séjour. Elle décrit la probabilité qu'aura une molécule entrant dans le volume de contrôle (par exemple la surface ${ }^{-} \mathrm{du}^{-}$'sol) au temps $\mathrm{t}^{\prime}$ de sortir (par exemple à une profondeur définie) entre les instants $\tau=t-t^{\prime}$ et $\tau+d \tau$. Elle est donc censée représenter l'effet de l'ensemble des processus (sans les expliciter) et de la nature des conditions initiales et aux limites sur le temps de séjour d'une substance dans un profil de sol.

Ce type d'approche, de nature non mécaniste a été utilisé avec des succès certains pour simuler des expériences in-situ, réalisées avec différentes substances $(\mathrm{Br}, \mathrm{Cl}$, Nitrates, pesticides). Pour plus de détails, on pourra notamment se reporter aux travaux de Jury (1982), JURY et al. (1986), WHITE et al. (1986) et à l'ouvrage de JURY et ROTH (1990) fort bien documenté.

II nous semble que cette approche peut constituer un modèle de gestion de site intéressant dans la mesure où il ne requiert qu'un nombre réduit de données. Cependant elle présente l'inconvénient inhérent à tous les modèles fondés sur le concept de fonction de transfert (classiquement utilisé en Hydrologie) : la nécessité d'un étalonnage spécifique aux conditions d'utilisation.

Toute modification du milieu et/ou des conditions aux limites oblige à l'établissement d'une nouvelle fonction.

\section{CONCLUSIONS ET SUGGESTIONS}

De cette brève analyse et d'autres précédemment publiées (ADDISCOTT et WAGENET, 1985 ; NIELSEN et al., 1986 ; GAUDET et VAUCLIN, 1987 ; WAGENET et al., 1988 ; VACHAUD et al., 1993), il est possible de dégager quelques conclusions et tendances, et d'avancer quelques suggestions.

1. La communauté scientifique dispose d'une pléthore de modèles et d'approches. A titre d'exemple, le tableau 2 en présente une liste, non exhaustive. Son examen montre une grande diversité dans leur degré de complexité et d'applicabilité à l'échelle de l'environnement. De nombreux modèles sont đéveloppés pour un besoin spécifique, et ne peuvent pas (ou difficilement) être utilisés dans des situations autres que celles pour lesquelles ils ont été développés. 
Tableau 2 Exemple de modèles disponibles.

Table 2 Some currently available models (drawn from DE COURSEY, 1991).

\begin{tabular}{|c|c|c|}
\hline Model Name & Author Reference & Use \\
\hline SLIM & Aoolscon, 1982 & Nitrate movement in the field \\
\hline SWAM & ALONSO and DE COURSEY, 1985 & $\begin{array}{l}\text { Small watershed scale-fertilizer, pesticide, } \\
\text { sediment \& general hydrology }\end{array}$ \\
\hline WATCROS & ASLYNG and HANSEN, 1982 & Water balance and crop production \\
\hline ANIMO & BERGHUUS et al, 1985 & Nitrogen modet \\
\hline \multirow[t]{2}{*}{ PULSE } & BERGSTROM et al, 1987 & Nitrate leaching \\
\hline & $\begin{array}{l}\text { BOESTEN, } 1986 \text { - } \\
\text { BURNS, } 1985\end{array}$ & $\begin{array}{l}\text { Pesticide movement } \\
\text { Nitrate leaching }\end{array}$ \\
\hline PRZM & CARSEL et al, 1984 & Solute leaching \\
\hline \multirow[t]{3}{*}{ TRANS CSM } & CHARDON, 1984 & Cadmium mobility \\
\hline & CLOTHEER etal, 1988 & Ammonium nitrate movernent \\
\hline & DAUTREBANDE and AGNEESENS, 1985 & Pesticide transport \\
\hline \multirow[t]{2}{*}{ TRANS } & DE SMEDT et al, 1981 & Solute movement (mobile/immobile water) \\
\hline & DE WILLIGEN et al, 1982 & Phosphate transport \\
\hline \multirow[t]{2}{*}{ PESTAN } & ENFiELD et al, 1982 & Pesticide transport \\
\hline & FERRARI and CUPERIUS, 1973 & Non-absorbed ion transport \\
\hline MORELN & GeNG et al, 1987 & Nitrate transport \\
\hline NITCROS & HANSEN and ASLYNG, 1984 & Nitrogen balance and crop products \\
\hline RZWOM & HEBSON and DE COURSEY, 1987 & $\begin{array}{l}\text { Mgmt. model-fertilizer, pesticide, solute and } \\
\text { transport in general }\end{array}$ \\
\hline WHNSIM & HUWE and VAN DER PLOEG, 1987 & Plant uptake \\
\hline SOILN & JOHNSSON et al, 1987 & Nitrogen dynamics \\
\hline BAM & JURY et al, 1983 & Transport of pesticides and other organics \\
\hline CREAMS & KNISEL, 1980 & Water quality (general) \\
\hline RENLEM & KRAGT and DE VRIES, 1987 & Solute transport-plant uptake waterflow \\
\hline GLEAMS & LEONARD et al, 1986 & Water quality (general) \\
\hline CALF & Nicholls et af, 1982 & Pesticide degradation and movement \\
\hline \multirow[t]{2}{*}{ CMLS } & NOFZIGER and HORNSBY, 1985 & A management tool for chemical movement \\
\hline & RICHTER et al, 1988 & Nitrate processes (general) \\
\hline SIMUL & RIEU, 1984 & Solute transport and precipitation \\
\hline COFARM & SCHAFFER et al, 1984 & Nitrogen and tillage mgmt at farm level \\
\hline NTRM & SCHAFFER and LARSON, 1987 & Nitrogen tillage, residue mgmt \\
\hline OPUS & FERREIRA and SMITH, 1990 & General hydrology-field scale \\
\hline \multirow[t]{2}{*}{ MOUSE } & STEENHUIS et al, 1987 & Pesticide transport \\
\hline & VAN DER ZEE and VAN RIEMSDLJK, 1986 & Phosphate transport \\
\hline \multirow[t]{2}{*}{ ONZAT } & VAN DRECHT, 1985 & Field/Lab treatment of non linearly sorbet solutes \\
\hline & WINIEN ef al, 1988 & Crop response to saltne water \\
\hline \multirow{2}{*}{ LEACHMP } & WAGENET anO HUISON, & 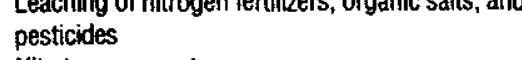 \\
\hline & ZANOT and DE WILIGEN, 1981 & Nitrate movement \\
\hline
\end{tabular}

2. La perception et la compréhension des phénomènes liés aux transferts couplés d'eau et de substances chimiques dans les sols partiellement saturés s'avèrent délicates. Prendre en compte les différents processus conduit généralement à introduire de nombreux paramètres qui font qu'un modèle, qui est nécessairement une schématisation de la réalité peut aisément s'ajuster aux données expérimentales sans nécessairement contribuer à une meilleure connaissance des mécanismes, ni servir de guide à de nouvelles recherches et d'indicateur d'actions pratiques à entreprendre. 
3. Malgré les progrès très notables réalisés ces vingt dernières années tant à l'échelle de la colonne de laboratoire, qu'à celle du terrain, plusieurs questions restent à l'heure actuelle sans réponses. Parmi celles-ci, on peut citer :

- Comment prendre en compte les chemins préférentiels qu'ils soient dûs à la présence de macroporosités ou à l'émergence d'instabilités hydrodynamiques? Les écoulements sont-ils toujours darciens?

- Comment modéliser au mieux les effets de la variabilité spatiale et temporelle des propriétés hydrodynamiques des sols sur le transport de substances chimiques? Alors que l'approche déterministe semble avoir montré ses limites, l'approche_stochastique est-elle la voie sur laquelle il faut s'engager?

- Comment coupler efficacement des sous-modèles physico-chimiques, géochimiques et biologiques aux modèles hydro-dispersifs?

- Comment améliorer les techniques et méthodes d'estimation in-situ des paramètres décrivant les phénomènes de transfert et de transport?

- Comment améliorer la prévision des conséquences à long terme de décisions prises à court terme ? De plus, un effort supplémentaire de recherche devrait semble-t-il porter sur i) les transferts dans les sols structurés et/ou gonflants dont la plupart sont fortement interactifs avec les éléments chimiques, ii) le transport en phase gazeuse en liaison notamment avec le devenir des substances volatiles, iii) la modélisation des bio-transformations.

4. En dehors d'un manque de connaissances sur certains aspects, il nous semble qu'une grande partie des verrous actuels vient de la propension des communautés scientifiques concernées à limiter leur domaine d'investigation et de vision, à leur propre discipline. Reprenons l'exemple de l'équation (2). Un examen de la littérature met en évidence les tendances suivantes:

- les pnysiciens du sol négligent très souvent les termes (a) (e) et (f) et utilisent des « traceurs " pour déterminer $D(\theta, q)$. Ils suggèrent des améliorations à l'équation résultante fondées par exemple sur une meilleure connaissance (indispensable par ailleurs) de la topologie interne du milieu poreux (i.e. le concept de phases fluides mobile et immobile).

- les chimistes du sol mettent l'accent sur les paramètres intervenant dans les termes (a) et (e) pour étudier les phénomènes d'échanges, les réactions chimiques, etc. Très souvent, ils négligent, par les essais en batch, les 3ème et 4ème termes représentatifs du transport diffusif et convectif respectivement.

- les microbiologistes du sol étudient essentiellement le 5ème terme de l'équation (2) en relation avec le développement et l'activité des populations microbiennes. De plus, ils choisissent des conditions expérimentales telles que les 3ème et 4ème termes soient négligés.

- les agronomes et physiologistes végétaux quant à eux s'intéressent au dernier terme, en liaison notamment avec la production ou la protection végétale.

Les progrès importants réalisés dans la description des équilibres entre phases, dans le développement des métrologies et des moyens informatiques permettent de lever certains verrous dès lors que l'on accepte de s'affranchir du poids des traditions. II s'agit d'adapter la fondamentalité de la démarche théorique au problème concret à résoudre en ne retenant à l'échelle considérée que les phénomènes prépondérants. 
A cet égard, une approche pluridisciplinaire apparaît indispensable, même si elle doit bousculer la sacro-sainte classification d'A. Comte!..

5. Une tâche importante à ne pas négliger est l'application des résultats de la recherche aux problèmes concrets de la gestion des ressources en eau et en sol en vue d'une meilleure protection de notre environnement. Trop peu de modèles sont réellement confrontés à des réalités de terrain. Dans cette optique, trois points au moins doivent mériter toute notre attention :

- l'impérieuse nécessité de développer d'une part des techniques d'observations et d'analyse performantes, d'autre part des expérimentations bien conçues en vue de déterminer les paramètres pertinents et d'obtenir des données pour qualifier ceš mödèles en fonction des objectifs pratiques poursuivis.

- l'établissement de critères quantitatifs de validation des modèles prenant en compte leurs incertitudes et qui soient clairement identifiés et universellement reconnus. Ce demier aspect apparaît important afin notamment de conduire des comparaisons objectives des performances des différents modèles.

- alors que le nombre de modèles et de codes numériques croît de façon quasi-exponentielle, il est temps de se poser la question suivante : la Communauté Scientifique doit-elle continuer à développer des modèles de plus en plus sophistiqués (voire spécifiques) ou doit-elle mettre laccent sur les expérimentations de terrain? La réponse n'est pas anodine pour le futur. En effet, les coûts informatiques ne cessant de décroitre et ceux liés aux activités expérimentales devenant prohibitifs, on constate une tendance mondiale à " observer les transferts d'eau et de substances chimiques au travers d'écrans d'ordinateurs! ”. Les modélisateurs doivent avoir conscience que sans des estimations fiables des paramètres et des validations bien conduites, leurs modèles apparaîtront plus comme un jeu intellectuel d'intérêt aca. démique, que comme des outils d'aide à la prise de décisions.

Lever les barrières entre expérimentation et modélisation, entre praticiens et théoriciens, entre les différentes disciplines devrait être une impérieuse obligation dans les années à venir. Puisse cette contribution et beaucoup d'autres renforcer l'idée de la nécessité de concilier ou de réconcilier en une préoccupation unique les études de l'écoulement et des interactions biophysico-chimiques, l'ensemble étant pris au sens large.

\section{RÉFÉRENCES BIBLIOGRAPHIQUES}

ABBOTT M.B., BATHURST J.C., CUNGE J.A., O'CONNELL P.E., RASMUSSEN J., 1986. An introduction to the European Hydrological System. 1 - History and philosphy of a physically based distributed modeling system and 2 - Structure of a physically- based distributed modeling system. $J$. Hydrol. 87, 45-77.

ADDISCOTT T.M., 1977. A simple computer model for leaching in structured soils. $J$. Soil Sci., 28, 554-563. 
ADDISCOTT T.M., 1982. Computer assessment of the $N$ status during winter and early spring. In " Assessment of the Nitrogen Status of the Soils ". T. Batey et al., Ed. University Press, Leuven : 15-26.

ADDISCOTT T.M., WAGENET R.J., 1985. Concepts of solute leaching in soils : a review of modeling approaches. J. Soil Sci., 36, 411-424.

ADOISCOTT T.P., HEYS P.J., WHITMORE A.P., 1986. Application of simple leaching models in heterogeneous soils. Geoderma, 38, 185-194.

ALONSO C.V., DECOURSEY D.G., 1985. Small Watershed Model. In D.G. DeCoursey (ed.), Proceedings of the Natural Resources Modeling Symposium, Pingree Park, CO, Oct. 16-21, 1983, USDAARS-30, 40-46.

ASLYNG H.C., HANSEN S., 1982. Water Balance and Crop Production Simulation. Model WATCROS for Local and Regional Application. The Royal Vet. and Agric. Univ. Copenhagen, $200 \mathrm{p}$.

BAJRACHARYA K., BARRY D.A., 1993. Mixing cell models for non linear equilibrium single species adsorption and transport. $J$. of Contaminant Hydrol., 12, 227-243.

BERGHUIJS J.T., RIJTEMA P.E., ROEST C.W.J., 1985. ANIMO, Agricultural Nitrogen Model, NOTA 1671, Inst. for Land and Water Management Research Wageningen.

BERGSTROM L., BRANDT M., GUSTAFSON A., 1987. Simulation of runoff and nitrogen leaching from two fields in Southern Sweden. Hydrol. Sci. J., 32, 191-205.

BIGGAR J.W., NIELSEN D.R., 1976. Spatial variability of the feaching characteristics of a field soil-water. Water Resour. Res., 12, 78-84.

BOESTEN J.J.T.J., 1986. Behavior of herbicides in soil: simulation of experimental assessment. Doct. Thesis, inst. for Pest. Res. Wageningen, 263 p.

BOLT G.H., 1979. Soil chemistry, physicochemical models. Elsevier, New-York, 479 pp.

BOND W.J., SMILES D.E., 1983. Influence of velocity on hydrodynamic dispersion during unsteady soil water flow. Soil Sci. Soc. Am. J., 47, 438-441.
BOND W.J., 1986. Velocity-dependent hydrodynamic dispersion during unsteady, unsaturated soil water flow: Experiments. Water Resour. Res., 22, 1881-1889.

BRESLER E., 1967. A model for tracing sait distribution in the soil profile and estimating the efficient combination of quality and quantity under varying field conditions. Soil Sci., 104, 227-233.

BRESLER E., DAGAN G., 1979. Solute dispersion in unsaturated heterogeneous soil at field scale. II-Applications. Soil Sci. Soc. Am. J., 43, 467-472.

BAESLEA E., DAGAN G., 1981. Convective and pore scale dispersive solute transport in unsaturated flow conditions. Water Resour. Res., 17, 1683-1693.

BRESLER E., DAGAN G., 1983. Unsaturated flow in spatially variable fields. 3 - Solute transport models and their application in two fields. Water Resour. Res., 19, 429 435.

BRUSSEAU M.L., RAO P.S.C., 1990. Modeling solute transport in structured soils : a review. Geoderma, 46, 169-192.

BURNS I.G., 1974. A model for predicting the redistribution of salts applied to fallow soils after excess rainfall or evaporation. $J$. Soil Sci., 25, 165-178.

BURNS I.G., 1975. An equation to predict the leaching of surface-applied nitrate. J. Agric. Sci. Cambridge, 85, 443-454.

BURNS I.G., 1976. Equations to predict the leaching of nitrate uniformily incorporated to a known depth or uniformly distributed throughout a soil profile. J. Agric. Sci. Cambridge, 86, 305-313.

BURNS I.G., 1980. A simple model for predicting the effects of leaching of fertilizer nitrate during the growing season on the nitrogen fertilizer needs of crops. $J$. Soil Sci., 31, 175-202.

BURNS I.G., 1985. An equation to predict the leaching of surface-applied nitrate. J. Agric. Sci. Cambridge, 85, 443-454.

CALVET R., TERCE M., ARVIEU J.C., 1980. Adsorption des pesticides par les sols et leurs constituants. III - Caractéristiques générales de l'adsorption des pesticides. Ann. Agron., 31, 239-257.

CAMERON KC., WILD A., 1982. Prediction of solute leaching under field conditions : An 
appraisal of three methods. J. Soil Sci., 33, 659-669.

CARNAHAN C.L., REMER J.S., 1984. Non equilibrium and equilibrium sorption with linear sorption isotherm during mass iransport through an infinite porous medium: some analytical solutions. $J$. Hydrol., 73, 227-258.

CARSEL R.F., MULKEY L.A., LORBER M.N., BASKIN L.B., 1984. A procedure for evaluating pesticide leaching threats to groundwater. Ecological Modeling, 30, 49-69.

CHARDON W.J., 1984. Mobility of cadmium in soil. (in Dutch). Doctorat Thesis, Wageningen Agricultural University, Serie Bodembescherming $n^{\circ} 36$, Staatsuitgeverij, s-Gravenhage, $200 \mathrm{p}$.

CHAISTENSEN S., SIMKINS S., TIEDJE J.M., 1990. Spatial variability in denitrification: dependence of activity centers in the soil environment. Soil Sci. Soc. Am. J., 54, 1608-1613.

CHRYSIKOPOULOUS C.V., KITANIDIS P.K., ROBERTS P.V., 1990. Analysis of onedimensional solute transport through porous media with spatially variable retardation factor. Water Resour. Res., 26, 437-446.

CLOTHIER B.E., 1990. Root Zone Processes and Water Quality : The impact of management. In D.G. DeCoursey (ed.), Proceedings International Symposium on Water Quality Modeling of Agricultural Non-Point Sources, June 19-23, 1988, Logan, UT, U.S. Dep. of Ag., Ag. Res. Serv., ARS-81, 659-674.

CLOTHIER B.E., SAUER T.T., GREEN S.R., 1988. The movement of ammonium nitrate into unsaturated soil during unsteady absorption. Soil Sci. Soc. Am. J., 52, 340345.

COASTS K.H., SMITH B.D., 1964. Dead-end pore volume and dispersion in porous media. Soc. Pet. Eng. J., 4, 73-84.

DAGAN G., RUSSO D., BRESLER E., 1990. Effect of spatial variability upon subsurface transport of solutes from nonpoint sources. In DG. DeCoursey (ed.), Proceedings International Symposium on Water Quality Modeling of Agricultural non-point sources, June 19-23, Logan, UT, U.S. Dep. of. Ag., Ag. Res. Serv., ARS-81, 523-548.
DAGAN G., BRESLER E., 1983. Unsaturated flow in spatially variable fields. 1 . Derivation of models of infiltration and redistribution. Water Resour. Res., 19 (2), 413-420.

DAUTREBANDE S., AGNEESENS J.P., 1985. Pévision du transfert des pesticides dans les sols. Aspects de Standardisation, Ed. Inra $n^{\circ}$ 31, 87-104.

DAVIDSON J.M., MC DOUGAL J.R., 1973. Experimental and predicted movement of three herbicides in water-saturated soil. $J$. Environ. Qual, 2, 428-433.

DECOURSEY D.G., ROJAS K.W., 1990. RZWQM - A model for simulating the movement of water and solutes in the root zone. In D.G. DeCoursey (ed.), Proceedings International Symposium on Water Quality Modeling of Agricultural non-point sources, June 19-23, 1988, Logan, UT, U.S. Dep. of Ag., Ag. Res. Serv., ARS-81, 813-822.

DECOURSEY D.G., 1991. Integrated quantity/quality modeling. Root zone leaching. In D.S. Bowles and P.E. O'Connell (eds), Recent advances in the modeling of hydrologic systems. Nato ASI Series, 345, 289-322.

DE WILLIGEN P., BERGSTROM L., GERRITSE R.G., 1990. Leaching models of the unsaturated zone, their potential use for management and planning. In D.G. DeCoursey (ed.), Proceedings International Symposium on Water Quality Modeling of Agricultural non-point sources, June 19-23, 1988, Logan, UT, U.S.Dep. of Ag., Ag. Res. Serv., ARS-81, 105-128.

DE WILLIGEN P., NEETESON J.J., 1985. Comparison of six simulation models for the nitrogen cycle in the soil. Fert. Res., 8, 157 171.

DE WILLIGEN P., RAATS P.A.C., GERRITSE R.G., 1982. Transport and fixation of phosphate in acid homogeneous soils. II Computer simulation. Agric. Environ., 7, 161-174.

ENFIELD C.G., CARSEL R.F., COHEN S.Z., PHAN T., WALTERS D.M., 1982. Approximating pollutant transport to ground water. Ground Water, 20, 711-722.

ENGESGAARD P., CHRISTENSEN T.H., 1989. A review of chemical solute transport modess. J. Cont. Hydrol., 17, 28-57.

FERRARI T.J., CUPERUS J.L., 1973. Dynamic simulation of vertical non-adsorbed anion transport. Plant Soil, 38, 425-438. 
FERREIRA V.A., SMITH R.E., 1900. OPUS, an advanced simulation model for nonpoint source pollution transport at the field scale. An Overview. In DG. DeCoursey (ed.), Proceedings International Symposium on Water Quality Modeling of Agricuttural non-point sources, June 19-23, Logan, UT, U.S. Dep. of Ag., Ag. Res. Serv., ARS-81, 823-834.

FOLORUNSO O.A., ROLSTON D.E., 1984. Spatial variability of field-measured denitrification gas flux. Soil Sci. Soc. Am. J., 48, 1214-1219.

FRISSEL M.J., POELSTRA P., 1967. Chromatic transport through soils. If - Column experiments with $\mathrm{Sr}$ - and $\mathrm{Ca}$ - isotopes. Plant Soil, 27, 20-32.

GAUDET J.P., JEGAT H., VACHAUD G., WIERENGA P.J., 1977. Solute transfer with exchange between mobile and stagnant water, through unsaturated sand. Soil Sci. Soc. Am. J., 41, 665-671.

GAUDET J.P., VAUCLIN M., 1987. Transfert de l'eau et de substances chimiques dans les sols partiellement saturés: de la colonne au milieu naturel. Colloque Int. " Impact de la physico-chimie sur l'étude, la conception et l'optimisation des procédés en milieu poreux naturel ", Nancy, Comptes-Rendus, 253-274.

GAMERDINGER A.P., WAGENET R.J., VAN GENUCHTEN M. Th., 1990. Application of two-sites/two-regions models for studying simultaneous non equilibrium transport and degradation of pesticides. Soil Sci. Soc. Am. J., 54, 957-963.

GENG Q.Z., GIRARD G., SOULARD Y., BLONDEL A., 1987. Modélisation du transfert de nitrates sur le bassin versant de la Noe-Séche-Bretagne. Rapport CIG. ENSMP-LHMRD, $30 \mathrm{p}$.

GRUNDMAN G.L., ROLSTON D.E., KACHANOSKI R.G., 1988. Fleld soil properties influencing the variability of denitrification gas fluxes. Soil Sci. Soc. Am. J., 52, 1351-1355.

HANSEN S., ASLYNG H., 1984. Nitrogen balance in crop production. Simulation model NITCROS. The Royal Vet. and Agric. Univ. Copenhagen, $113 \mathrm{p}$.

HAVERKAMP R., PARLANGE J.Y., RENDON L., KUTILEK M., 1988. Infiltration under ponded conditions. Infiltration equations tested for parameter time-dependence and predictive use. Soil Sci., 145, 317-329.
HAYOT C., 1992. Transfert de solutés en milieu poreux agrégé. Expérimentation et tentative de validation d'un modèle mécaniste. Doctorat de I'Université $L$. Pasteur, Strasbourg.

HEBSON C.S., DECOURSEY D.G., 1987. A model for assessing agricultural management impact on ground water quality. Jour. Env. Sci. and Health, 22, 18-34.

HUTSON J.L., WAGENET R.J., 1993. A pragmatic field-scale approach for modeling pesticides. J. Environ. Qual., 22, 494-499.

HUWE B., VAN DER PLOEG R.R., 1987. Erfahrungen und problem bie der simulation des $\mathrm{N}$-Haushalts Verschieden Genutzter Standarte Baden-Wurttembergs. Mitteilgn. Disch. Bodenkundl. Gesselsch., 55/, 181-187.

JENSEN J.R., 1984. Potassium dynamics in soll during steady flow. Soil Sci., 138, 285293.

JOHNSSON $H$., BERGSTROM L., JANSSON P.E., PAUSTIAN K., 1987. Simulated nitrogen dynamics in a layered agricultural soil. Agric. Ecosystems Environ., 18, 333356.

JURY W.A., 1982. Simulation of solute transport using a transfer function model. Water Resour. Res., 18, 363-368.

JURY W.A., 1982. Simulation of solute transport using a transfer function model. Water Resour. Res., 18, 363-368.

JURY W.A., STOLZY L.H., 1982. A field test of the transter function model for predicting solute transport. Water Resour. Res., 18, 369-375.

JURY W.A., SPENCER W.F., FARMER W.J., 1983. Model for assessing behavior of pesticides and other trace organics using benchmark properties. I - Description of model. J. Environ. Qual., 12, 558-564.

JURY W.A., SPOSITO G., WHITE R.E., 1986. A transfer function model of solute transport through soil. I - Fundametal concepts. Water Resour. Res., 22, 243247.

JURY W.A., ROTH K., 1990. Transfer function and solute movement through soil. Theory and applications. Birkhauser Verlag, Basel (Ed.), 226 p.

KNIGHT J.H., 1983. Infiltration equations from exact and approximate solutions of 
Richards'equation. In "Advances in Infiltration ", Am. Soc. of Agricultural Engineers (Ed.), 21-33.

KNISEL W.G., 1980. CREAMS : A field-scale model for chemicals, runoff and erosion from agricultural management systems. U.S. Dept. of Agric., Conservation Research, Report $n^{\circ} 26,640 \mathrm{p}$.

KOOKANA R.S., SCHULLER R.D., ALYMORE L.A.G., 1993. Simulation of simazine transport through soil columns using time-dependent sorptior data measured under flow conditions. $J$. of Contaminant Hydrol., 14, 93-115.

KRAGT J.F., DE VRIES W., 1987. Onderzoek naar effecten van Mestbeperking op de Nitraat Uitspoeling in water-wingebieden in Overysel. 1 - Beschrijving van RENLEM. Stiboka Rapport no 1935, Wageningen.

LAPIDUS L., AMUNDSON N.R., 1952. Mathematics of absorption in beds. 6 - The effects of longitudinal diffusion in ion exchange and chromatographic columns. J. Phys. Chem., 56, 984-988.

LAUDELOUT H., DUFFEY J.E., SHETA T.H. (1979). Ionic equilibria in semi-arid soils. 14th Coll. of the int. Potash. Inst. on " Soils in Mediterranean type climates and their potential yield". Sevilla, Spain, Proceedings, 99-114.

LEONARD R.A., KNISEL W.G., STILL D.A., 1986. Groundwater loading effects of agricultural management system. Trans. ASAE Paper, 86, 2498-2511.

MCBRATNEY A.B, WEBSTER R., 1983. How many observations are needed for regional estimation of soil properties? Soil Sci., $135,177-183$.

MANTOGLOU A., GELHAR L.W., 1987. Stochastic modeling of large-scale transient unsaturated flow systems. Water Resour. Res., 23, 37-67.

NICHOLLS P.H., WALKER A., WALKER R.J., 1982. Measurement and simulation of the movement and degradation of atrazine and metribuzin in a fallow soil. Pesticide Science, 13, 484-494.

NIELSEN D.R., BUGGAR J.W., 1961. Miscible displacement in soils. 1 - Experimental information. Soil Sci. Soc. Am. Proc., 25, 1-5.

NIELSEN D.R., BIGGAR J.W., ERTH K.T., 1973. Spatial variability of field measured soil-water properties. Hilgardia, 42, 215260.

NIELSEN D.R., VAN GENUCHTEN M. Th., BIGGAR J.W., 1986. Water flow and solute transport processes in the unsaturated zone. Water Resour. Res., 22, 89S-108S.

NKEDI-KIZZA P., BIGGAR J.W., VAN GENUCHTEN M.Th. and OTHERS, 1983. Modeling tritium and chloride 36 transport through an aggregated oxisol. Water Resour. Res., 19, 691-700.

NKEDI-KIZZA P., BIGGAR J.W., VAN GENUCHTEN M.Th. and OTHERS, 1984. On the equivalence of two conceptual models for describing ion-exchange during transport through on aggregated oxisol. Water Resour, Res., 20, 1123-1130.

NOFZIGER D.L., HORNSBY A.G., 1985. A microcomputer-based management tool for chemical movement in soil. Applied Agricultural Research, 1, 50-56.

NOVY-QUADRI M., 1993. Transferts de solutés dans les sols saturés et non saturés. Application au pentachlorophénol. Doctorat de I'Université Joseph-Fourier, Grenoble.

PANDEY R.N., GUPTA S.K., 1978. Equations to predict leaching of solube salts in saline soils. J. Abric. Sci. Cambridge, 91, 131-134.

PARKIN T.B., ROBINSON J.A., 1989. Stochastic models of denitrification. Appl. Environ. Microbiol., 55, 72-77.

PARKIN T.B., SHELTON D.R., 1992. Spatial and temporal variability of carbofuran degradation in soil. J. Environ. Qual., 21, 672-678.

PARKIN T.B., 1993. Spatial variability of microbial processes in soil. A review. $J$. Environ. Qual., 22, 409-417.

PASSOURIA J.B., 1971. Hydrodynamic dispersion in aggregated media. Soil Sci., 11, 339-344.

PECK A., 1983. Field variability of soil physical properties. Adv. Imig., 2, 189-221.

PHILIP J.R., 1968. Diffusion, dead-end pores and linearized absorption in aggregated media. Aust. J. Soil Res., 6, 21-30.

PIVER W.T., LINDSTROM F.T., 1991. Numerical methods for describing chemical transport in the unsaturated zone of the subsurface. J. of Contaminant Hydrol., 8 , 243-262. 
RAATS P.A.C., 1978. Convective transport of solutes by steady flows. I - General laboratory. Agric. Water Manag., 1, 201 218.

REINIGER P., BOLT G.H., 1972. Theory of chromatography and its application to cation exchange in soils. Neth. J. Agric. Sci, 20, 301-313.

RICHTER J., NUSKE A., BOHMER M., WEHRMANN J., 1980. Simulation of nitrogen mineralization and transport in Loess-Parabrownearthes : Plots experiments. Plant Soil, 54, 329-337.

RICHTER J., SCHARPF H.C., WEHRMANN J., 1978. Simulation der winterlichen nitratverlagerung un böden. Plant Soil, 49, 381-393.

RICHTER J., KERSEBAUM K.C., UTERMANN J., 1988. Modeling of the Nitrogen Regime of Arable fieled soils. In D.S. Jenkinson and K.A. Smith (Eds.), Nitrogen Efficiency in Agricultural soils and the efficient use of fertilizer nitrogen. CEC Seminar.

RIEU M., 1984. Simulation numérique du transfert vertical des solutés et des équilibres d'échange et de précipitation dans les sols calcaires gypseux. Sci. Geol. Bull., 37, 253-266.

ROSE C.W., CHICHESTER F.W., WILLIAMS J.R., RITCHIE J.T., 1982. Application of an approximate analytic method of computing solute profiles with dispersion in soils. $J$. Environ. Qual., 11, 151-155.

ROTH K., JURY W.A., 1993. Modeling the transport of solutes to groundwater using transfer functions. J. Environ. Qual., 22, 487-493.

ROTH K., JUAY W.A., FLÜLHER H., ATTINGER W., 1991. Transport of chloride through an unsaturated field soil. Water Resour. Res., 27, 2533-2541.

RUSSO D., BRESLER E., 1980. Scaling soil hydraulic properties of a heterogeneous field. Soil Sci. Soc. Am. J., 44, 681-684.

RUSSO D., 1991. Stochastic analysis of simulated vadose zone solute transport in a vertical cross section of a heterogeneous soil during non-steady infiltration. Water Resour. Res., 27, 267-283.

SARDIN M., KREBS R., SCHWEICH D., 1986. Transient mass-transport in the presence of non linear physico-chemical interaction law progressive modeling and appropriate experimental procedures. Geoderma, 38 , 115-130.

SARDIN M., SCHWEICH D., LEIJ F.J., VAN GENUCHTEN M.Th., 1991. Modeling the non equilibrium transport of linearly solutes in porous media : a review. Water Resour. Res., 27, 2287-2307.

SCOTTER D.R., 1978. Preferential solute movement through larger soil voids. I-Some computations using simple theory. Aust. J. Soil Res., 16, 257-267.

SELIGMAN N.G., VAN KEULEN H., 1981. PAPRAN. A Simulation model of annual pasture production limited by rainfall and nitrogen. In Frissef M.J. and Veen J.A., eds. Simulation of Nitrogen Behaviour of Soil-Plant Systems. Wageningen PUDOC, 192-221.

SHAFFER M.J., LARSON W.E., 1987. NTRM, a soil-crop simulation model for nitrogen, tillage and crop-residue management. U.S. Deptartment of Agriculture, Conservation Research, Report 34-1, 103 p.

SHAFFER M.J., SWAN J.B., JOHNSON M.R., 1984. Coordinated farm and research management (COFARM) data system for soils and crops. J. Soil and Water Conser., $39,320-324$.

SHARMA M.L., LUXMOORE R.J., 1979. Soil spatial variability and its consequences on simulated water balance. Water Resour. Res., 15, 1567-1573.

DE SMEDT F., WIERENGA P.J., 1978. Approximate analytical solution for the solute flow during infiltration and redistribution. Soil Sci. Soc. Am. J., 42, 407-412.

DE SMEDT F., WIERENGA P.J., 1984. Solute transport through columns of glass beads. Water Resour. Pes., 20, 225-232.

SMITH R.E., PARLANGE J.Y., 1978. A parameter efficient hydrologic infiltration model. Water Resour. Res., 14, 533-538.

SMILES D.E., PERROUX K.M., ZEGELIN S.J., RAATS P.A.C., 1981. Hydrodynamic dispersion during constant rate absorption of water by soil. Soil Sci. Soc. Am. J., 45, 453-458.

SPOSITO G., JURY W.A., GUPTA V.K., 1986a. Fundamental problems in the stochastic convection-dispersion model of solute transport in aquifers and field soils. Water Resour. Res., 22, 77-88. 
SPOSITO G., WHITE R.E., DARRAH P.R., JURY W.A., 1986b. A transfer function model of solute transport through soil. 3 The convection-dispersion equation. Water Resour. Res., 22, 255-262.

STEENHUIS T.S., PACENKA S., PORTER K.S., 1987. MOUSE. A management model for evaluating groundwater contamination from diffuse surface sources aided by computer graphics. Applied Agricultural Research, 34, 14-28.

TABOR J.A., WARRICK A.W., MYYER'S D.E., PENNINGTON D.A., 1985. Spatial variability of nitrate in irrigated cotton : Il. Soil nitrate and correlated variables. Soil Sci. Soc. Am. J., 49, 390-394.

TANJI K.K., DONEEN L.D., FERRY G.V., AYERS R.S., 1972. Computer simulation analysis on reclamation of salt -affected soils in San Joaquin Vatley-California. Soil Sci. Soc. Am. Proc., 36, 127-133.

TERKELTROUB R.W., BABCOCK K.L., 1971. A simple method for predicting salt movement through soil. Soil Sci., 111, 182187.

VACHAUD G., VAUCLIN M., ADDISCOTT T.M., 1993. Solute transport in the vadose zone : a review of moels. In A. Avogadro et R.C. Ragaini (Eds.), Technologies for Environmental Cleanup: soil and groundwater, 157-185.

VALOCCHI A.J., 1985. Validity of the local equilibrium assumption for modeling sorbing solute transport through homogeneous soils. Water Resour. Res., 21, 808820.

VALOCCHI A.J., STREET R.L., ROBERTS P.V., 1981. Transport of ion-exchanging solutes in groundwater: chromatographic theory and field simulation. Water Resour. Res., 17, 1517-1527.

VAN DER ZEE S.E.A.T.M., VAN RIEMSDIJK W.H., 1986. Transport of phosphate in a heterogeneous field. Transport in Porous Media, 1, 339-359.

VAN DRECHT G., 1985. Toetsing van model ONZAT m. b. v. de Meetgegevens Uit Het Hydrologisch Proefgebied * Hupselve Beek ". RIVM Report 847211001, $80 \mathrm{p}$.

VAN EIJKEREN J.C.H., LOCH P.G., 1984. Transport of cationic solutes in sorbing porous media. Water Resour. Res., 20, 714-718.
VAN GENUCHTEN M.Th., DAVIDSON J.M., WIERENGA P.J., 1974. An evaluation of kinetic and equilibrium equations for the prediction of pesticide movement in porous media. Soil Sci. Soc. Am. J., 38, 29-35.

VAN GENUCHTEN M.Th., WIERENGA P.J., 1976. Mass transfer studies in sorbing porous media. I-Analytical solutions. Soil Sci. Soc. Am. Proc., 38, 29-35.

VAN GENUCHTEN M.Th., WIERENGA P.J., 1977. Mass transfer studies in sorbing porous media. III-Experimental evaluation with 1,4,5 T. Soil Sci. Soc. Am. J., 41, 278285.

VAN GENUCHTEN M.Th., ALVES W.J., 1982. Analytical solutions of the one-dimensional convective-dispersive solute transport equation. Tech. Bufl. 1661. Dept. Agric. Washington D.C., $151 \mathrm{p}$.

VAN GENUCHTEN M.Th., GARELICK S.M., YEH W.W.G., 1990. Application of parameter estimation techniques to solute transport studies. In D.G. DeCoursey (Ed.), Proceedings International Symposium on Water Quality Modeling of Agricultural nonpoint sources, June 19-23, Logan, UT, U.S. Dep. of Ag., Ag. Res. Serv., ARS-81, 731-754.

VAN HOORN J.W., 1981. Salt movement leaching efficiency and leaching requirement. Agric. Water Manag., 4, 409-428.

VAN DER MOLEN W.H., 1956. Desalinization of saline soils as a column process. Soil Sci., 81, 19-27.

VAN VEEN J.A., FRISSEL M.J., 1981. Simulation model of the behavior of $N$ in soil. PUDOC, 126-144, Wageningen.

VAUCLIN M., 1983. Méthodes d'études de la variabilité spatiale des propriétés d'un sol. In * Variabilité spatiale des processus de transferts dans les sols. ", Ed. Les Colloques de I'INRA, $n^{\circ}$ 15, 9-43.

VAUCLIN M., IMBERNON J., VACHAUD G., DANCETTE C., 1983. Description expérimentale et modélisation stochastique des transferts par mise en facteur d'échelle des propriétés hydrodynamiques des sols. Proc. IAEA ISM 267/24, 103-124.

VILLERMAUX J., 1981. The chromatographic reactor. In Percolation Processes : Theory and Applications. Sijthoff and Noordhoff, Rockville, Maryland (eds.). 
VILLERMAUX J., VAN SWAAY W.P.M., 1969 Modèle représentatif de la distribtion des temps de séjour dans un réacteur semiinfini à dispersion axiale avec zones stagnantes. Chem. Eng. Sci., 24, 10071011.

VINTEN A., FRENKEL H., SHALHEVET H., 1988. Validation of a modified steady state model of crop response to saline water irrigation under transient conditions. Irrigation Sci., 29, 35-48.

VOLZ M., 1986. Variabilité spatiale des propriétés physiques du sol en milieu naturel. Thèse de l'Ecole Nationale Supérieure Agronomique, Montpellier.

WAGENET R.J., BIGGAR J.W., NIELSEN D.R., 1977. Tracing the transformations of urea fertilizer during leaching. Soil Sci. Soc. Am. J., 41, 896-902.

WAGENET R.J., HUTSON J.L., 1987. LEACHM, Leaching estimation and chemistry model, a process based model of water and solute movement, transformation, plant uptake and chemical reactions in the unsaturated zone. Water Resour. Inst. 2, Cornell University, Ithica, N.Y.

WAGENET R.J., SHAFFER M.J., GREEN P.E., 1990. Predictive approaches for leaching in the unsaturated zone. In D.G. DeCoursey (Ed.), Proceedings Internatio. nal Symposium on Water Quality Modeling of Agricultural non-point sources, June 19-23, Logan, UT, U.S. Dep. of Ag., Ag. Res. Serv., ARS-81, 63-80.
WAGENET R.J., RAO B.K., 1983. Description of nitrogen movement in the presence of spatially-variable soil hydraulic properties. Agric. Water Manag., 6, 227-242.

WARRICK A.W., MULLEN G.J., NIELSEN D.R., 1977. Scaling field-measured soil hydraulic properties using a similar media concept. Water Resour. Res., 13, 355-362.

WARRICK A.W., AMOOZEGAR-FARD A., 1979. Infiltration and drainage calculations using spatially scaled hydraulic properties. Water Resour. Res., 15, 1116-1120.

WARRICK A.W., MULLEN G.J., NIELSEN D.R., 1977. Predictions of the soil water flux based upon field measured soil water properties. Soil Sci. Soc. Am. J. 41, 14-19.

WARRICK A.W., NIELSEN D.R., 1980. Spatial variability of soil physical properties in the field. In D. Hillel ed. Applications of Soil Physics, 319-344, Academic, Orlando, Fla.

WHITE R.E., DYSON J.S., HAIGH R.A., JURY W.A., SPOSITO G., 1986. A transfer function model of solute transport through soil. 2 - lliustrative applications. Water Resour. Res., 22, 248-254.

ZANDT P.A., DE WILLIGEN P., 1981. Simulatie van de stikstofverdeling in de grond in winter en voorjaar. Inst. Bodemuruchtbaameid, Maren (Gn), 4-81.

ZHANG H., HAAN C.T., NOFZIGER D.L. 1993. An approach to estimating uncertainties in modeling transport of solutes through soils. J. of Contaminant Hydrol., $12,35-50$. 